\title{
Pre-eclampsia and childhood asthma
}

\author{
Maria C. Magnus ${ }^{1,2,3}$, Siri E. Håberg ${ }^{1}$, Per Magnus ${ }^{4}$, Anders Engeland ${ }^{1,5}$,

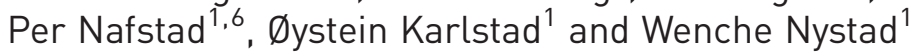

Affiliations: ${ }^{1}$ Domain for Mental and Physical Health, Norwegian Institute of Public Health, Oslo, Norway. ${ }^{2}$ MRC Integrative Epidemiology Unit, University of Bristol, Bristol, UK. ${ }^{3}$ School of Social and Community Medicine, University of Bristol, Bristol, UK. ${ }^{4}$ Domain for Health Data and Digitalization, Norwegian Institute of Public Health, Oslo, Norway. ${ }^{5}$ Dept of Global Public Health and Primary Care, Medical Faculty, University of Bergen, Bergen, Norway. ${ }^{6}$ Dept of Community Medicine, Medical Faculty, University of Oslo, Oslo, Norway.

Correspondence: Maria C. Magnus, Domain for Mental and Physical Health, Norwegian Institute of Public Health, P.O. Box 4404 Nydalen 0403 Oslo, Norway. E-mail: Maria.Christine.Magnusafhi.no

ABSTRACT Studies of pre-eclampsia and childhood asthma are conflicting, and none have performed a formal mediation analysis of preterm birth.

We examined the association between pre-eclampsia and asthma at 7 years using national registries, including all births in Norway from 1999 to 2006 ( $\mathrm{n}=406907)$, and a subsample of children in the Norwegian Mother and Child Cohort Study $(\mathrm{MoBa})(\mathrm{n}=45028)$ using log-linear regression. We performed a mediation analysis of preterm birth, and a sibling comparison to evaluate unobserved confounding.

There was a positive association between pre-eclampsia and asthma in the registry study, with an adjusted relative risk of 1.31 (95\% CI 1.22-1.41), but not in MoBa, which had an adjusted relative risk of 1.19 (95\% CI 0.99-1.44). The odds ratios for the direct effect not mediated through preterm birth and the indirect effect in the registry linkage were 1.19 (95\% CI 1.10-1.29) and 1.12 (95\% CI 1.11-1.14), respectively. The sibling comparison indicated no association between pre-eclampsia and asthma (adjusted OR 1.07, 95\% CI 0.87-1.33).

In this large study, which used different datasets and analytic approaches, there was little evidence for an association between pre-eclampsia and childhood asthma. The association was weak and largely explained by pre-term birth and confounders shared by siblings.

@ERSpublications

Pre-eclampsia shows no strong evidence for an association with childhood asthma at 7 years http://ow.ly/GoQ4302XGV3

Editorial comment in: Eur Respir J 2016; 48: 1541-1544.

This article has supplementary material available from erj.ersjournals.com

Received: April 032016 | Accepted after revision: July 30 2016 | First published online: Oct 062016

Support statement: The data collection in the Norwegian Mother and Child Cohort Study is supported by the National Institutes of Health (National Institute of Environmental Health Sciences contract number N01-ES-75558, National Institute of Neurological Disorders and Stroke grant no.1 UO1 NS 047537-01 and grant no.2 UO1 NS 047537-06A1) and the Norwegian Research Council/FMGE (grant number 151918/S10). This work was also supported by the Norwegian Research Council (grant number 221097 to W. Nystad). Funding information for this article has been deposited with the Open Funder Registry.

Conflict of interest: None declared.

Copyright OERS 2016 


\section{Introduction}

Asthma is the most common chronic disease in children, and is characterised by reversible airway obstruction and inflammation [1]. There is a genetic predisposition in the development of asthma [2]. However, despite decades of research, there are few established environmental causes, but pregnancy and early childhood are recognised as crucial time periods for the developing airways and immune system [3].

Pre-eclampsia is characterised by pregnancy-induced hypertension in combination with proteinuria, and occurs in between $2 \%$ and $8 \%$ of pregnancies [4]. Early-onset pre-eclampsia can affect placental development, with a subsequent reduced blood flow to the fetus [5]. Furthermore, pre-eclampsia often results in an earlier delivery, as delivery is the only intervention that will reverse the condition completely. Both preterm birth and low birth weight are associated with childhood asthma [6-8].

A few studies have examined the association between pre-eclampsia and childhood asthma in school age or later [9-12], one of which reported a positive association [10]. Two other studies have evaluated the association between pre-eclampsia and childhood wheezing phenotypes and found a positive association $[13,14]$. As far as we know, none of the previous studies conducted a formal mediation analysis to quantify the magnitude of the association between pre-eclampsia and childhood asthma mediated through preterm birth. Only one study performed a sibling comparison analysis [10] to evaluate the potential role of unobserved confounding at sibling level [15]. Most previous studies had a relatively modest sample size, and therefore limited power to evaluate pre-eclampsia as a risk factor for asthma.

The heterogeneity and inconsistent results of previous studies indicate that we need to address this research question using different methodological approaches and carefully assess the potential influence from confounding and mediating factors.

The objectives of the current study were 1) to examine the association between pre-eclampsia and childhood asthma, and 2) to evaluate whether preterm birth might mediate the association between pre-eclampsia and childhood asthma. We evaluated these objectives in both a national registry-based study and a large prospective pregnancy cohort, including detailed questionnaire information for additional potential confounding factors. The registry-based study included all births in Norway between January 1999 and July 2006, and the large number of children was the basis for using a sibling design.

\section{Methods}

\section{Study subjects}

The registry-based study included information from the Medical Birth Registry of Norway (MBRN), the Norwegian Prescription Database (NorPD) and the Norwegian Education Database. This linkage encompassed all children born in Norway and registered in the MBRN between January 1999 and July 2006 ( $\mathrm{n}=443300$ ). Only live-born singletons with a valid national identification number, a birth weight of $500 \mathrm{~g}$ or more, a gestational age of 22 weeks or longer, and who were alive and living in Norway on their eighth birthday were eligible for the current study (figure 1a). This left 406907 children in the analysis.

The Norwegian Mother and Child Cohort Study $(\mathrm{MoBa})$ is a prospective pregnancy cohort administered by the Norwegian Institute of Public Health [16, 17]. MoBa recruited pregnant women between 1999 and 2008, at approximately 18 weeks' gestation. The participation rate was $41 \%$. Mothers could participate in the cohort with more than one pregnancy, resulting in a cohort of 95000 mothers and 114500 children. All participants gave a written informed consent. In this study, we included children with information from questionnaires administered at 18 and 30 gestational weeks, in addition to questionnaires administered when the child was 6 months, with a birth weight of $500 \mathrm{~g}$ or more, a gestational age of 22 weeks or longer, and who had reached 7 years by April 1, 2013 (figure 1b). This left 45028 children born between January 2000 and March 2006 in the analysis. Data from MoBa was linked to the MBRN and the NorPD.

The Norwegian Data Inspectorate and the Regional Committees for Medical and Health Research Ethics of South/East and West Norway approved this study.

\section{Pre-eclampsia}

In Norway, pregnant women carry a standardised chart to all antenatal examinations during pregnancy. At the time of delivery, the midwife transfers information from this chart to the MBRN notification form, which is mandatory for all births. The MBRN has five tick boxes relevant to pre-eclampsia: 1) HELLP syndrome (haemolysis, elevated liver enzymes and low platelet count); 2) eclampsia; 3) early pre-eclampsia (diagnosed before 34 weeks); 4) mild pre-eclampsia; and 5) severe pre-eclampsia. We defined pre-eclampsia (yes/no) as a registration of any of these conditions in the MBRN. We combined information on gestational age at birth with information on pre-eclampsia into four mutually exclusive categories (neither, pre-eclampsia only, preterm birth only and pre-eclampsia with preterm birth). Preterm birth was defined as a gestational age of less than 37 weeks. 

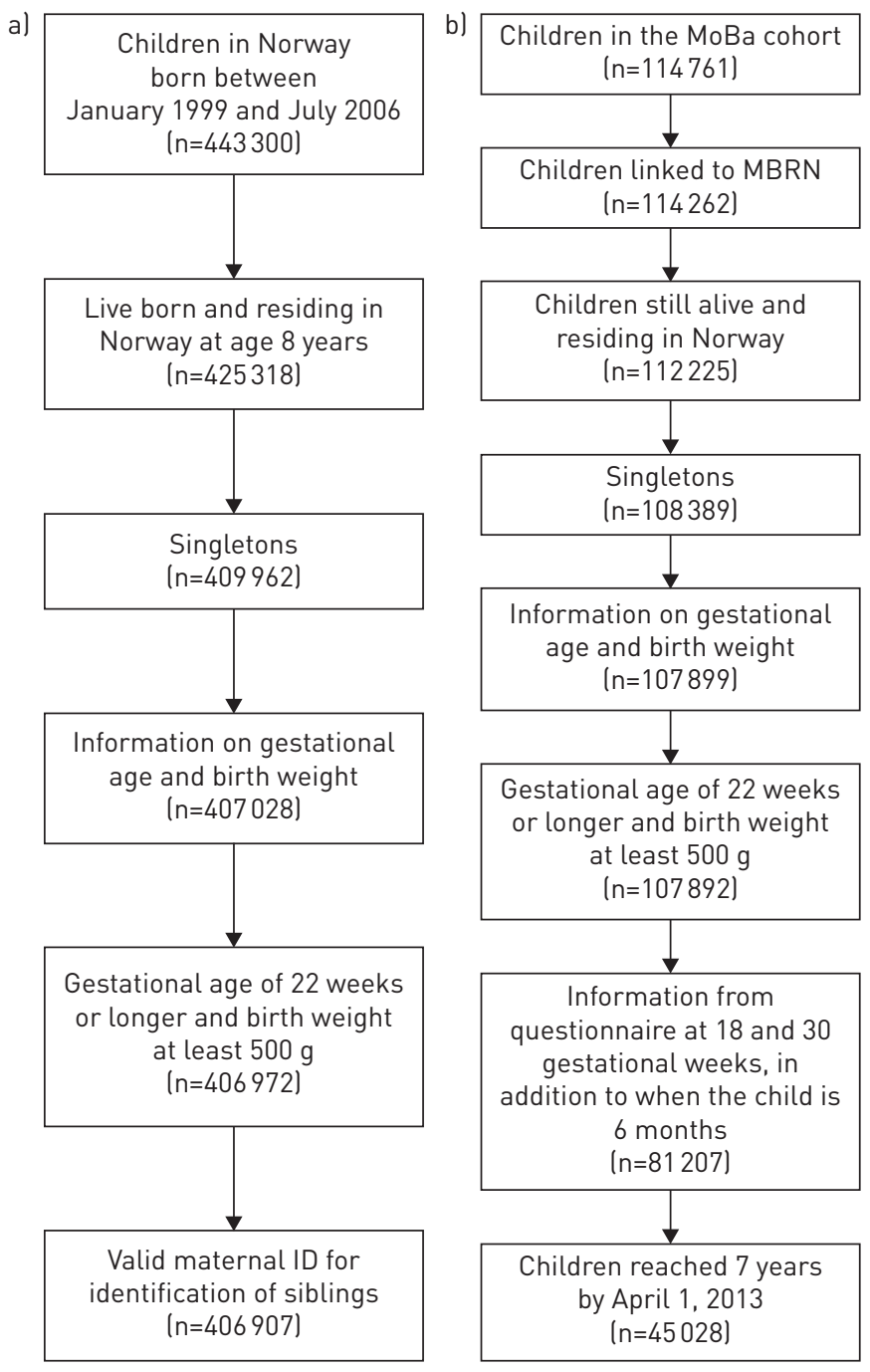

FIGURE 1 Illustration of sample selection. a) Registry-based study. b) The Norwegian Mother and Child Cohort Study (MoBa). MBRN: Medical Birth Registry of Norway.

\section{Childhood asthma}

The child's asthma status was defined by evaluating dispensed asthma medications as registered in the NorPD. The NorPD contains information on all dispensed prescriptions from all Norwegian pharmacies since January 2004. Asthma medications included inhaled short- and long-acting beta(2)-agonists (R03AC), inhaled corticosteroids (R03BA), fixed-dose combinations of inhaled beta(2)-agonists and corticosteroids (R03AK), and leukotriene antagonists (R03DC). A child was defined as having asthma at 7 years if there was at least one dispensed prescription for asthma medications in the past 12 months in addition to at least one second dispensed prescription within the 12 months after the first. In a secondary analysis within the MoBa cohort, we defined children as having asthma if the mother reported in the questionnaire answered when the child was 7 years old that the child was doctor diagnosed with asthma and also had either current symptoms or had used asthma medications in the past 12 months.

\section{Covariates}

We used information on characteristics that could potentially influence the association of interest. Child characteristics included sex (male/female). Maternal characteristics included age at delivery (entered as a continuous variable), parity (categorised into primiparous, 1, 2 and 3 or more), education (categorised into less than secondary school, secondary school, up to 4 years of university and 4 or more years of university), maternal asthma (categorised as yes/no) and smoking during pregnancy (categorised as yes/ no). Additional information from questionnaires in MoBa included maternal pre-pregnancy body mass index (BMI; entered as a continuous variable).

\section{Statistical analysis}

We did descriptive analyses showing the distribution of pre-eclampsia by the covariates. The further analysis was done in several steps. First, we used national registry data and evaluated the association between 
pre-eclampsia and the child's risk of asthma using log-binomial regression models, reporting relative risks and 95\% confidence intervals, accounting for the dependency between siblings by using robust cluster variance estimation. The multivariable analyses adjusted for the maternal age, parity, education, maternal asthma and the child's sex. Additional adjustment for maternal smoking during pregnancy was explored among the $75 \%$ of children for whom this information was available in the registry. Second, we repeated the analysis in the subsample of children participating in the MoBa cohort. In the multivariable analyses using MoBa data, we further adjusted for pre-pregnancy BMI in addition to the characteristics mentioned above. Third, we conducted a secondary analysis in MoBa using a questionnaire-based asthma definition as the outcome.

Fourth, we evaluated the independent and combined effect of preterm birth and pre-eclampsia on asthma development, and subsequently calculated the relative excess risk due to interaction, before moving on to a formal mediation analysis. Figure 2 shows the theoretical framework underlying our mediation analysis. The mediation analysis was performed by a logistic decomposition of the total effects into indirect and direct effects using the ldecomp command in the Stata software (Statacorp, College Station, TX, USA) [18]. We also explored conventional multivariable adjustment for preterm birth as an alternative approach to obtain an estimate of the direct effects of pre-eclampsia. Last, we used the national registry data and conducted an analysis of sibling pairs discordant for asthma using conditional logistic regression analysis, reporting odds ratios and $95 \%$ confidence intervals. This approach compares the distribution of pre-eclampsia between sibling pairs discordant for asthma.

The amount of missing covariate information was generally low $(<2 \%)$. Nevertheless, we explored the robustness of the results using multiple imputation by chained equations, imputing a total of 20 datasets. The statistical significance level was 5\%. The analysis was conducted using Stata version 14 (Statacorp).

\section{Results}

Distribution of pre-eclampsia by maternal and child characteristics

The prevalence of pre-eclampsia was $4 \%$, while $5 \%$ of children had asthma at 7 years. The prevalence of pre-eclampsia decreased with maternal age, parity, educational level and smoking, while it increased with maternal pre-pregnancy BMI (table 1). Furthermore, there was a higher prevalence of pre-eclampsia among mothers with asthma and among mothers delivering preterm (table 1).

\section{Pre-eclampsia and asthma at 7 years}

There was a positive association between pre-eclampsia and asthma at 7 years in the registry study, with an adjusted relative risk of 1.31 (95\% CI 1.22-1.41), but not in $\mathrm{MoBa}$, with an adjusted relative risk of 1.19 (95\% CI 0.99-1.44) (table 2). The difference in the strength of the association in the registry study and the MoBa cohort was mostly explained by additional adjustment for maternal pre-pregnancy BMI in MoBa. The adjusted relative risk was 1.27 (95\% CI 1.06-1.53) without adjustment for pre-pregnancy BMI. There was no change in the observed associations after exclusion of mothers with pre-existing hypertension before pregnancy (supplementary table E1). The multiple imputation analysis also gave similar results (supplementary table E2). Furthermore, there was no difference in the observed associations among primiparous and multiparous mothers (supplementary table E3). In MoBa, the magnitude of the association between pre-eclampsia and asthma at 7 years was larger and only statistically significant when using the maternal report of doctor-diagnosed asthma at 7 years as an outcome (supplementary table E4).

\section{Combined effects of pre-eclampsia and preterm birth on asthma at 7 years}

Around $4 \%$ of children were born preterm by mothers who did not have pre-eclampsia, $3 \%$ of children were born at term to mothers who had pre-eclampsia, while $1 \%$ of children were born preterm to mothers who had pre-eclampsia. There was a positive association between being born at term to mothers with pre-eclampsia and asthma at 7 years in the registry-based study (adjusted relative risk 1.21, 95\% CI 1.11-1.32) but not in MoBa (adjusted relative risk 1.01, 95\% CI 0.80-1.28) (table 2). There was some indication in MoBa that the association

FIGURE 2 Theoretical framework. Maternal background characteristics include maternal age, parity, education, body mass index, asthma and smoking during pregnancy.

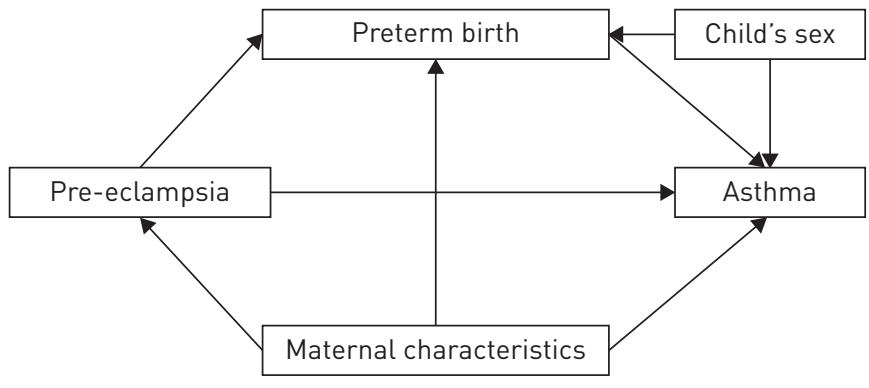


TABLE 1 Distribution of pre-eclampsia by maternal and child characteristics

Characteristics
Registry linkage ${ }^{\#}$

n $\%$ pre-eclampsia
MoBa"

n $\%$ pre-eclampsia

\begin{tabular}{|c|c|c|c|c|}
\hline \multicolumn{5}{|l|}{ Pre-eclampsia } \\
\hline No & 390578 & & 43281 & \\
\hline Yes & 16329 & 4.0 & 1747 & 3.9 \\
\hline \multicolumn{5}{|l|}{ Maternal age at delivery years } \\
\hline$<25$ & 70123 & 4.9 & 4996 & 5.5 \\
\hline $25-29$ & 137239 & 4.1 & 15457 & 4.3 \\
\hline $30-34$ & 135788 & 3.5 & 17300 & 3.2 \\
\hline $35+$ & 63757 & 3.9 & 7275 & 3.4 \\
\hline \multicolumn{5}{|l|}{ Maternal parity } \\
\hline Nulliparous & 164683 & 5.9 & 19511 & 5.6 \\
\hline 1 & 146081 & 2.8 & 16045 & 2.6 \\
\hline 2 & 68174 & 2.6 & 7407 & 2.4 \\
\hline $3+$ & 27969 & 2.7 & 2065 & 3.0 \\
\hline \multicolumn{5}{|l|}{ Maternal education } \\
\hline Less than secondary school & 77340 & 4.2 & 3789 & 4.5 \\
\hline Secondary school & 148773 & 4.4 & 14479 & 4.4 \\
\hline Up to 4 years of university & 137854 & 3.9 & 18387 & 3.6 \\
\hline More than 4 years of university & 29950 & 3.1 & 8206 & 3.3 \\
\hline Missing & 12990 & 2.7 & 167 & 5.4 \\
\hline \multicolumn{5}{|l|}{ Maternal asthma } \\
\hline No & 389995 & 4.0 & 43199 & 3.8 \\
\hline Yes & 16912 & 5.3 & 1829 & 5.2 \\
\hline \multicolumn{5}{|c|}{ Maternal smoking at start of pregnancy } \\
\hline No & 268177 & 4.1 & 39868 & 4.0 \\
\hline Yes & 72409 & 3.4 & 4968 & 2.8 \\
\hline Missing & 66321 & 4.2 & 192 & 2.1 \\
\hline \multicolumn{5}{|l|}{ Maternal pre-pregnancy BMI } \\
\hline Underweight $(<18.5)$ & NA & & 1253 & 2.4 \\
\hline Normal weight (18.5-24.9) & NA & & 28421 & 2.7 \\
\hline Overweight (25-29.9) & NA & & 9865 & 5.2 \\
\hline Obese $(\geqslant 30)$ & NA & & 4238 & 8.7 \\
\hline Missing & NA & & 1251 & 4.6 \\
\hline \multicolumn{5}{|l|}{ Year of birth } \\
\hline 1999 & 55155 & 4.3 & & \\
\hline 2000 & 54974 & 4.2 & 1102 & 4.5 \\
\hline 2001 & 52630 & 4.1 & 3265 & 4.2 \\
\hline 2002 & 51643 & 4.3 & 6878 & 4.3 \\
\hline 2003 & 52861 & 3.7 & 10060 & 3.6 \\
\hline 2004 & 53459 & 3.7 & 10632 & 3.9 \\
\hline 2005 & 53347 & 3.8 & 11828 & 3.7 \\
\hline 2006 & 32838 & 3.9 & 1263 & 3.6 \\
\hline \multicolumn{5}{|l|}{ Child gender } \\
\hline Male & 208643 & 4.1 & 22975 & 3.9 \\
\hline Female & 198264 & 4.0 & 22053 & 3.8 \\
\hline \multicolumn{5}{|l|}{ Preterm birth } \\
\hline No & 385458 & 3.2 & 42997 & 3.1 \\
\hline Yes & 21449 & 18.0 & 2031 & 19.5 \\
\hline
\end{tabular}

There were significant differences in the distribution of pre-eclampsia by all maternal and child characteristics $(p<0.003)$ except for child's sex $(p=0.109$ in the registry linkage and $p=0.639$ in MoBa). MoBA: Norwegian Mother and Child Cohort Study; BMI: body mass index. ${ }^{\#}: \mathrm{n}=406907 ;{ }^{\text {巾 }} \mathrm{n}=45028$.

between pre-eclampsia and preterm birth in combination with asthma was greater than the independent associations seen for these two exposures (table 2 and supplementary table E4). However there was no statistically significant excess relative risk due to interaction.

The mediating effect of preterm birth on the association between pre-eclampsia and asthma at 7 years

Pre-eclampsia was around six times more prevalent among those born preterm (table 1), and preterm birth was also a risk factor for asthma (table 2). We therefore evaluated the potential indirect effect of 
TABLE 2 Relative risks of asthma at 7 years in children born to mothers with pre-eclampsia

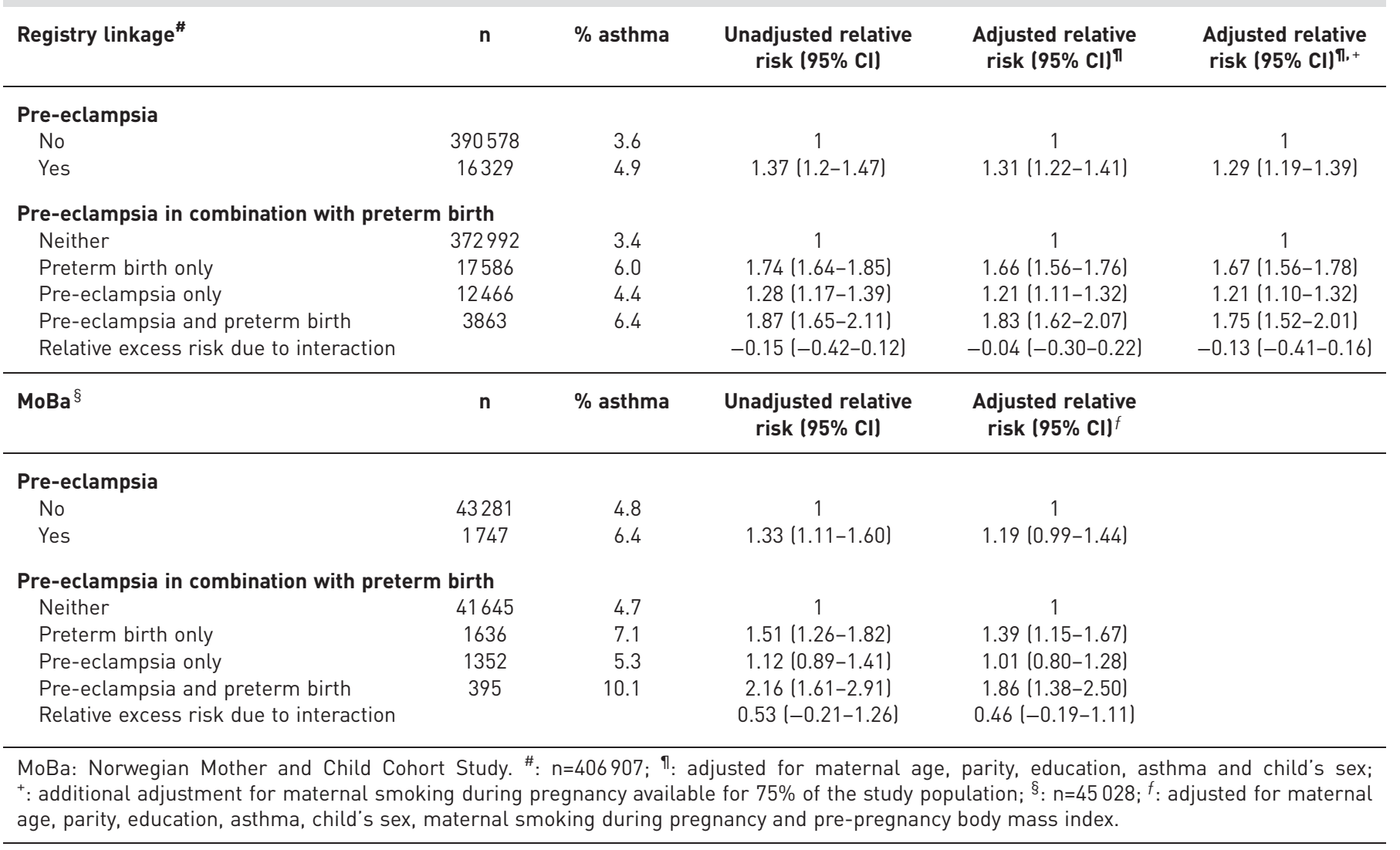

pre-eclampsia on asthma mediated through preterm birth. The results from the mediation analysis indicated a significant direct effect of pre-eclampsia on childhood asthma in the registry-based study (adjusted OR 1.19, 95\% CI 1.10-1.29), while the indirect effect was 1.12 (95\% CI 1.11-1.14) (table 3). There was no direct effect in MoBa (adjusted OR 1.11, 95\% CI 0.89-1.37) (table 3). When adjusting for preterm birth as a covariate, the results yielded an adjusted relative risk of 1.17 (95\% CI 1.10-1.27) in the registry linkage and 1.10 (95\% CI 0.91-1.33) in MoBa. Secondary analyses evaluated the combined indirect effect of preterm birth, low birth weight and delivery by caesarean section (supplementary table E5). The results of the direct effect not mediated through these three pregnancy outcomes was OR 1.11 (95\% CI 1.04-1.18) in the registry linkage and OR 1.03 (95\% CI 0.83-1.27) in MoBa.

TABLE 3 Logistic regression model of the direct and indirect effects through preterm birth of pre-eclampsia on asthma at 7 years

Adjusted OR (95\% Cl)

\begin{tabular}{|c|c|}
\hline \multicolumn{2}{|l|}{ Registry linkage } \\
\hline \multicolumn{2}{|l|}{ Component } \\
\hline Direct effect & $1.19(1.10-1.29)^{\pi}$ \\
\hline Indirect effect & $1.12(1.11-1.14)^{9}$ \\
\hline Total effect & $1.34(1.25-1.44)^{9}$ \\
\hline \multicolumn{2}{|l|}{$\mathrm{MoBa}^{+}$} \\
\hline \multicolumn{2}{|l|}{ Component } \\
\hline Direct effect & $1.11(0.89-1.37)^{\S}$ \\
\hline Indirect effect & $1.09(1.05-1.14)^{\S}$ \\
\hline Total effect & $1.21(0.98-1.49)^{\S}$ \\
\hline \multicolumn{2}{|c|}{$\begin{array}{l}\text { MoBa: Norwegian Mother and Child Cohort Study. }{ }^{\#}: \mathrm{n}=406907 ;{ }^{\Uparrow} \text { : adjusted for maternal age, parity, education, } \\
\text { asthma and child's sex; }{ }^{+}: \mathrm{n}=45028 ; \AA \text { : adjusted for maternal age, parity, education, asthma, child's sex, } \\
\text { maternal smoking during pregnancy and pre-pregnancy body-mass index. }\end{array}$} \\
\hline
\end{tabular}


Sibling pair analysis of pre-eclampsia and asthma at 7 years

There were a total of 5923 sibling groups (of two or more children) with at least two siblings discordant for asthma. The sibling pair analysis indicated no significant association between pre-eclampsia and childhood asthma (adjusted OR 1.07, 95\% CI 0.87-1.33) (table 4).

\section{Discussion}

In this large-scale prospective study, we observed a positive association between pre-eclampsia and school-age asthma. Our findings indicate that this association could be largely explained by a mediating effect of preterm birth. Furthermore, we cannot exclude the possibility of unobserved confounding due to characteristics that are shared by siblings.

\section{Strengths and limitations of the study}

The different methodological approaches in this study have their strengths and limitations and when interpreting the results these should be taken into consideration. The overall analysis in the registry linkage had the best power, but is likely to be hampered by some unobserved confounding. We therefore explored the association in the subset of individuals participating in $\mathrm{MoBa}$ with more detailed information on potential confounding factors, and added a registry-based sibling comparison analysis. Results from the MoBa analyses might be influenced by selection bias due to the participation rate [17], and the sibling comparison conducted within the registry linkage had limited power due to the modest number of discordant sibling pairs available for analysis. However, there was sufficient power to detect a significant association between preterm birth and asthma in the sibling pair analysis. Our study also has additional limitations. Using the MBRN to classify pre-eclampsia might have resulted in misclassification, as indicated by a validation study [19]. The prevalence of asthma at 7 years was similar when the asthma definition was based on the prescription registry (4.8\%) or maternal report through questionnaires $(5.8 \%)$, which strengthens the reliability of an asthma prevalence of around $5 \%$ at age 7 . However, this is a lower prevalence than in some other European countries. For example, the estimated prevalence of asthma at approximately 7 years is $11 \%$ in the Avon Longitudinal Study of Parents and Children (ALSPAC) in the UK [20], while current asthma at 6 years was only reported for $3 \%$ in the Generation $\mathrm{R}$ study in the Netherlands [21]. It was also not possible to distinguish between full versus half siblings. The results of the sibling pair analysis might therefore not account for shared genetic predisposition to a full extent as would be expected based on a comparison of known full siblings. Although we did not know the ethnicity of the study participants, excellent Norwegian language skills were a requirement for participation in $\mathrm{MoBa}$ and the overwhelming majority of MoBa participants are assumed to be of European descent.

The mediation analysis conducted in this study assumed no residual or unobserved confounding for the association between pre-eclampsia and asthma, pre-eclampsia and preterm birth, or preterm birth and asthma. A mediation analysis can also cause a spurious association due to collider stratification bias [22]. We adjusted for a wide range of maternal characteristics, but we cannot exclude the possibility that our observed associations still could be influenced by residual confounding. The results should therefore be interpreted with caution. Furthermore, the direct effect is to be interpreted as the residual association not mediated through preterm birth.

\section{Comparison with previous studies}

Four previous studies have examined the association between pre-eclampsia and childhood asthma [9-12]. A large Danish registry-based study observed an incidence rate ratio of 1.19 (95\% CI 1.15-1.24) between

TABLE 4 Sibling pair analysis of associations between pre-eclampsia in combination with preterm birth with asthma at 7 years ( $n=5923$ discordant sibling groups)

Unadjusted OR $(95 \% \mathrm{CI}) \quad$ Adjusted OR $(95 \% \mathrm{CI})^{\#}$

\begin{tabular}{lcc}
\hline Pre-eclampsia & & 1 \\
No & 1 & 1.07 (0.87-1.33) \\
Yes & $1.16(0.94-1.42)$ & 1 \\
Pre-eclampsia in combination with preterm birth & & $1.55(1.28-1.87)$ \\
$\quad$ Neither & 1 & $1.09(0.86-1.38)$ \\
Preterm birth only & $1.57(1.32-1.88)$ & $1.17(0.79-1.73)$ \\
Pre-eclampsia only & $1.17(0.94-1.46)$ & \\
Pre-eclampsia and preterm birth & $1.30(0.90-1.89)$ & \\
\hline
\end{tabular}

\#: Adjusted for maternal age, parity, education, asthma and child's sex. 
pre-eclampsia and asthma [10]. In contrast, a Norwegian historical cohort study $(\mathrm{n}=617)$ indicated no association between pre-eclampsia and asthma at 10 years of age (OR 0.72, 95\% CI 0.19-2.77) for severe pre-eclampsia [9]. Similarly, findings from ALSPAC indicated no association between pre-eclampsia and asthma at 7 years (adjusted OR 1.23, 95\% CI 0.80-1.88) [12]. A large Norwegian registry study of all live births from 1967 to $1993(\mathrm{n}=1548429)$, which linked the MBRN to the National Insurance Administration Register, indicated that pregnancy complications (including pre-eclampsia) could represent risk factors for childhood asthma [11]. The current study further contributes information to these previous studies by conducting a formal mediation analysis of the indirect effects due to preterm birth.

\section{Interpretation of findings}

Our finding of a stronger association of pre-eclampsia in combination with preterm birth on the risk of asthma is likely to reflect the underlying severity of pre-eclampsia. Of the three studies of pre-eclampsia and school-age asthma that adjusted for gestational age, one found no association even before adjustment [12], one indicated that the association was attenuated and became non-significant after multivariable adjustment for gestational age [10], while the third found no association after adjustment for a number of factors without specifically examining the influence of adjustment for gestational age [9]. Our study is therefore the first to attempt to quantify the indirect effects of pre-eclampsia on childhood asthma explained by preterm birth, and indicated evidence of a direct effect of pre-eclampsia on childhood asthma in the registry linkage [23]. As expected, the estimate of the combined indirect effect of preterm birth, low birth and delivery by caesarean section was larger than observed for preterm birth alone. However, a significant direct effect of pre-eclampsia not mediated through these three pregnancy outcomes was still found in the registry analyses.

A potential explanation for a direct effect between pre-eclampsia and asthma not mediated through poor placental function leading to poor intra-uterine growth and preterm birth is the influence of immunological mechanisms. Maternal immune tolerance is important to ensure a healthy pregnancy outcome, and pre-eclampsia is hypothesised to be partly due to a maladaptation of this necessary immune tolerance during pregnancy [24-26]. Studies are beginning to unravel the potential interactions between the innate and the adaptive immune system that are required to maintain a healthy pregnancy [24]. One might speculate that the failure of the necessary immunological adaptions during pregnancy could also have consequences for the offspring's development of immune-related diseases such as asthma.

The fact that we observed a direct effect of pre-eclampsia on asthma development not mediated through preterm birth in the registry linkage and not in MoBa could be explained by the additional adjustment for pre-pregnancy BMI in MoBa. Overweight and obesity are well-known risk factors for pre-eclampsia [27, 28]. This might be explained by the systemic inflammation and subsequent immunological changes observed among those who are obese or overweight [29]. We have previously found maternal obesity during pregnancy to increase the risk of wheezing in infancy [30], and thus maternal BMI might confound the association between pre-eclampsia and childhood asthma.

A sibling comparison allows an evaluation of the likelihood that unobserved background characteristics shared by siblings are influencing the observed associations [15]. Only one previous study performed a sibling comparison when evaluating the association between pre-eclampsia and childhood asthma [10]. In line with our findings, this previous study indicated no association between pre-eclampsia and asthma, supporting the notion that the weak direct effect observed in the overall analysis in the registry-based study might be influenced by confounding due to unobserved background characteristics shared by siblings. However, it is important to acknowledge that any sibling comparison is more susceptible to confounding by unobserved background characteristics not shared by siblings [15].

\section{Conclusion}

In this large study, which used different datasets and analytic approaches, there was little evidence for an association between pre-eclampsia and childhood asthma. The association was weak and largely explained by pre-term birth and confounders shared by siblings.

\section{Acknowledgements}

We are grateful to all the families participating in the Norwegian Mother and Child Cohort Study.

\section{References}

Asher I, Pearce N. Global burden of asthma among children. Int J Tuberc Lung Dis 2014; 18: 1269-1278.

2 Lim RH, Kobzik L, Dahl M. Risk for asthma in offspring of asthmatic mothers versus fathers: a meta-analysis. PloS One 2010; 5: e10134.

3 Wegienka G, Zoratti E, Johnson CC. The role of the early-life environment in the development of allergic disease. Immunol Allergy Clin North Am 2015; 35: 1-17.

4 Duley L. The global impact of pre-eclampsia and eclampsia. Semin Perinatol 2009; 33: 130-137. 
Huppertz B. Placental origins of preeclampsia: challenging the current hypothesis. Hypertension 2008; 51: 970-975.

6 Jaakkola JJ, Ahmed P, Ieromnimon A, et al. Preterm delivery and asthma: a systematic review and meta-analysis. J Allergy Clin Immunol 2006; 118: 823-830.

7 Sonnenschein-van der Voort AM, Arends LR, de Jongste JC, et al. Preterm birth, infant weight gain, and childhood asthma risk: a meta-analysis of 147,000 European children. J Allergy Clin Immunol 2014; 133: 1317-1329.

$8 \mathrm{Mu} \mathrm{M}, \mathrm{Ye} \mathrm{S}$, Bai MJ, et al. Birth weight and subsequent risk of asthma: a systematic review and meta-analysis. Heart Lung Circ 2014; 23: 511-519.

9 Byberg KK, Ogland B, Eide GE, et al. Birth after preeclamptic pregnancies: association with allergic sensitization and allergic rhinoconjunctivitis in late childhood; a historically matched cohort study. BMC Pediatr 2014; 14 : 101.

10 Liu X, Olsen J, Agerbo E, et al. Maternal preeclampsia and childhood asthma in the offspring. Pediatr Allergy Immunol 2015; 26: 181-185.

11 Nafstad P, Samuelsen SO, Irgens LM, et al. Pregnancy complications and the risk of asthma among Norwegians born between 1967 and 1993. Eur J Epidemiol 2003; 18: 755-761.

12 Shaheen SO, Macdonald-Wallis C, Lawlor DA, et al. Hypertensive disorders of pregnancy, respiratory outcomes and atopy in childhood. Eur Respir J 2015; 47: 156-165.

13 Rusconi F, Galassi C, Forastiere F, et al. Maternal complications and procedures in pregnancy and at birth and wheezing phenotypes in children. Am J Respir Crit Care Med 2007; 175: 16-21.

14 Zugna D, Galassi C, Annesi-Maesano I, et al. Maternal complications in pregnancy and wheezing in early childhood: a pooled analysis of 14 birth cohorts. Int J Epidemiol 2015; 44: 199-208.

15 Frisell T, Öberg S, Kuja-Halkola R, et al. Sibling comparison designs: bias from non-shared confounders and measurement error. Epidemiology 2012; 23: 713-720.

16 Magnus P, Irgens LM, Haug K, et al. Cohort profile: the Norwegian Mother and Child Cohort Study (MoBa). Int J Epidemiol 2006; 35: 1146-1150.

17 Nilsen RM, Vollset SE, Gjessing HK, et al. Self-selection and bias in a large prospective pregnancy cohort in Norway. Paediatr Perinat Epidemiol 2009; 23: 597-608.

18 Buis ML. Direct and indirect effects in a logit model. Stata J 2010; 10: 11-29.

19 Klungsoyr K, Harmon QE, Skard LB, et al. Validity of pre-eclampsia registration in the medical birth registry of Norway for women participating in the Norwegian mother and child cohort study, 1999-2010. Paediatr Perinat Epidemiol 2014; 28: 362-371.

20 Hoskin-Parr L, Teyhan A, Blocker A, et al. Antibiotic exposure in the first two years of life and development of asthma and other allergic diseases by $7.5 \mathrm{yr}$ : a dose-dependent relationship. Pediatr Allergy Immunol 2013; 24: $762-771$.

21 den Dekker HT, Sonnenschein-van der Voort AM, Jaddoe VW, et al. Breastfeeding and asthma outcomes at the age of 6 years. The Generation R Study. Pediatr Allergy Immunol 2016; 27: 486-492.

22 Wilcox AJ, Weinberg CR, Basso O. On the pitfalls of adjusting for gestational age at birth. Am J Epidemiol 2011; 174: $1062-1068$

23 Naimi AI, Kaufman JS, MacLehose RF. Mediation misgivings: ambiguous clinical and public health interpretations of natural direct and indirect effects. Int J Epidemiol 2014; 43: 1656-1661.

24 Hsu P, Nanan RK. Innate and adaptive immune interactions at the fetal-maternal interface in healthy human pregnancy and pre-eclampsia. Front Immunol 2014; 5: 125.

25 Redman CW, Sargent IL. Immunology of pre-eclampsia. Am J Reprod Immunol 2010; 63: 534-543.

26 Wilczynski JR. Immunological analogy between allograft rejection, recurrent abortion and pre-eclampsia-the same basic mechanism? Hum Immunol 2006; 67: 492-511.

27 Young OM, Twedt R, Catov JM. Pre-pregnancy maternal obesity and the risk of preterm preeclampsia in the American primigravida. Obesity 2016; 24: 1226-1229.

28 Vinturache A, Moledina N, McDonald S, et al. Pre-pregnancy body mass index (BMI) and delivery outcomes in a Canadian population. BMC Pregnancy Childbirth 2014; 14: 422.

29 Lyons CL, Kennedy EB, Roche HM. Metabolic inflammation-differential modulation by dietary constituents. Nutrients 2016; 8: pii: E247.

30 Haberg SE, Stigum H, London SJ, et al. Maternal obesity in pregnancy and respiratory health in early childhood. Paediatr Perinat Epidemiol 2009; 23: 352-362. 